\title{
Movement variability response to change in the rate of hopping
}

\author{
ABbigail L. Fietzer ${ }^{1,2 *}$, YUMiKo KOYAMA ${ }^{3,4}$, KORNELIA KULIG ${ }^{1}$ \\ ${ }^{1}$ Division of Biokinesiology and Physical Therapy, University of Southern California, Los Angeles, CA, USA. \\ ${ }^{2}$ Department of Physical Therapy, Mount Saint Mary's University, Los Angeles, CA, USA. \\ ${ }^{3}$ Department of Human Health Sciences, Kyoto University, Kyoto, Japan. \\ ${ }^{4}$ Department of Tokyo Physical Therapy, Faculty of Medical Sciences, Teikyo University of Science, Tokyo, Japan
}

\begin{abstract}
Movement variability is often considered undesirable, but growing evidence demonstrates positive aspects of variability. During unipedal hopping, control of limb stiffness and limb length are paramount. Purpose: The purpose of this study was to compare two methods of measuring movement variability that provide information at the task level, and their capacities to illuminate the neuromotor control system's response to change in hopping rate. Methods: The typical task-level movement variability measure of the standard deviation of vertical limb length was compared to uncontrolled manifold analysis. We examined the relationship between change scores in deviation from spring-mass model-type behavior and these two variability measures for the shift from typical $(2.3 \mathrm{~Hz})$ to slow $(1.7 \mathrm{~Hz})$ hopping. Results: The change scores for deviation from spring-mass model-type behavior and vertical limb length standard deviation demonstrated no correlation ( $p=0.784, R=0.051)$. In contrast, the change scores for deviation from spring-mass model-type behavior and the uncontrolled manifold analysis measure demonstrated a moderate correlation $(p=0.004, R=0.502)$. Conclusions: Uncontrolled manifold analysis considers not just variability in the sense of error, but illustrates how the neuromotor control system distributes movement variability into performance-irrelevant and performance-destabilizing subspaces. As such, this type of analysis may be more effective at illuminating global control aspects of movement variability than the typical variability measure of limb length standard deviation.
\end{abstract}

Key words: uncontrolled manifold, spring mass model, segmental coordination

\section{Introduction}

Movement variability is often considered the failure of an imperfect human control system, and, therefore, something to be minimized. However, movement variability is found to a surprising degree even in elite athletes [2], [4]. Variability tends to decrease with progression from novice to moderate skill level, but increase with progression from moderate skill to expertise [2], [10], [16], [19]. Furthermore, altered variability is associated with pathology in a variety of injury types and activities [3], [10], [11], [14]. While some of these studies link pathology with excessive variability, others link pathology with insufficient variability.

Findings of decreased variability in pathological populations point to the positive roles of variability.
Variability may enable multiple successful performance strategies and make the performer adaptable to small changes in task, equipment, or personal state, and even protect against injury [4], [10], [11], [14]. Most variability studies employ single-joint or dual-joint, or endpoint-only, measures of variability. Such measures provide a magnitude of variability, but no information about quality - whether it promotes or detracts from performance consistency.

Uncontrolled manifold (UCM) analysis parses variability into performance-irrelevant $\left(V_{\mathrm{UCM}}\right)$ and performance-destabilizing $\left(V_{\mathrm{ORT}}\right)$ subspaces, providing a distinction in movement variability quality not offered by most other measures of variability [23]. It is plausible that $V_{\mathrm{UCM}}$ (vs. $V_{\mathrm{ORT}}$ ) plays the positive roles of providing adaptability and protection against injury without disrupting performance. This assertion is consis-

\footnotetext{
* Corresponding author: Abbigail L. Fietzer, Department of Physical Therapy, Mount Saint Mary's University, 10 Chester Place, Los Angeles, CA 90007. Phone: 001-323-240-5418, e-mail: afietzer@msmu.edu

Received: July 2nd, 2019

Accepted for publication: September 19th, 2019
} 
tent with previous findings of a smaller proportion of $V_{\mathrm{UCM}}$ (vs. $V_{\mathrm{ORT}}$ ) variability in elders and persons with neurological disorders compared to younger or healthy counterparts [15], [20]. UCM analysis provides a promising tool to explore individual responses to perturbations or small changes within a task potentially associated with injury risk.

For UCM analysis, variability is measured at two levels. The first is a relatively microscopic examination of variability at the elemental contributor level. This microscopic level typically represents measures of kinematic variability. The second is a relatively macroscopic examination of variability at the task level. This macroscopic level typically represents measures of outcome variability. The elemental contributors may coordinate so that variability in one element cancels out variability in another. This cancelling-out promotes task level measure consistency, and is deemed performance-irrelevant variability $\left(V_{\mathrm{UCM}}\right)$. In contrast, the elemental contributors may fail to coordinate so that variability in one element is not countered by variability in another. This lack of coordination results in task level measure inconsistency, and is deemed performance-destabilizing variability $\left(V_{\mathrm{ORT}}\right)$.

The UCM method provides context to the control system's manner of distributing variability at the elemental level, whether it contributes to or detracts from task-level variability. Such detailed content is not available with basic movement variability measures, such as the standard deviation of vertical limb length across hopping trials $\left(V L L_{\mathrm{SD}}\right)$. While numerous studies examining movement variability from the perspective of single-joint or end-point standard deviation (akin to $V L L_{\mathrm{SD}}$ ) and others - using the UCM method have been conducted, the authors are unaware of any study comparing these differing movement variability perspectives using the same data set.

This study employs unipedal hopping, a naturally repetitive movement and tightly-controlled proxy for the more ecologically relevant bouncing gait of running. These types of bouncing gait are classically modeled by a spring-mass system $\left(m a_{V}+k \Delta L_{V}=m g\right)$, which accurately predicts all major mechanical parameters despite its apparent oversimplification of the entire lower limb into a spring [5]. The spring-mass model highlights the importance of spring compression $\left(\Delta L_{V}\right)$ control, prompting the use of vertical limb length variability as the task-level variability parameter examined in this study. Although not required for UCM analysis, elemental variables that have a straightforward mapping onto the task level variable are highly desirable [23]. For this study, elemental variables of foot-to-floor, ankle and knee local joint-coordinate sagittal plane intersegmental angles were chosen because they have a straightforward mapping onto the task level variable of vertical limb length (Fig. 1).

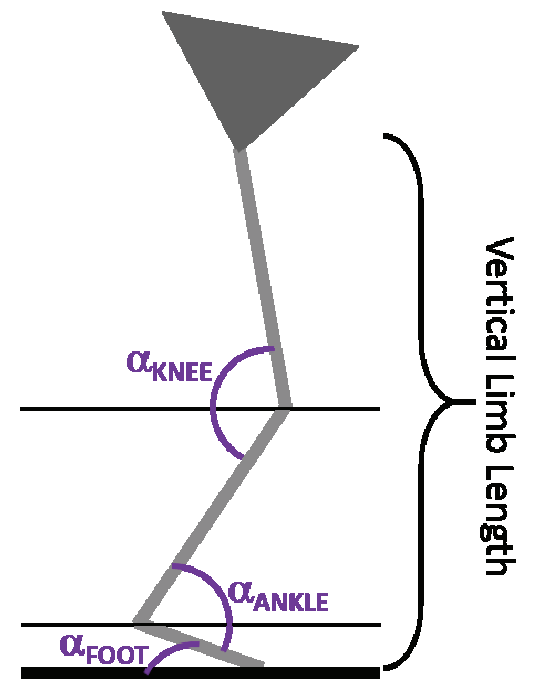

Fig. 1. Vertical limb length model for UCM analysis. The relationship between the elemental variables (sagittal plane foot-to-floor, and ankle and knee intersegmental angles) and the task-level variable (vertical limb length)

Hopping at rates slower than typically preferred presents a challenge to spring-mass model-type behavior, particularly with regards to maintaining linear spring stiffness $(k)$ [5]. However, maintenance of spring-mass model-type behavior has been demonstrated even in the presence of severe perturbations [7], [17]. Change in adherence to spring-mass model-type behavior is used as a representative of the degree of control strategy alteration in response to changing the hopping rate for this study, and is compared to change in the movement variability measures.

The purpose of this study is to compare the relationship between two different methods of measuring movement variability and change in adherence to spring-mass model-type behavior in response to altered hopping rate. The first method is the basic movement variability measure of the standard deviation of vertical limb length across hopping trials $\left(V L L_{\mathrm{SD}}\right)$. The second method is uncontrolled manifold analysis (UCM) of the degree to which coordination between variability in foot, shank and thigh positioning contributes to stabilization of vertical limb length across hopping trials. Due to the interest in response to change in hopping rate, within-participant changescores are the primary data analyzed. Correlations between change scores for each movement variability type, and change in adherence to spring-mass modeltype behavior (alteration in control strategy) are explored. 


\section{Materials and methods}

\section{Participants}

Thirty-four healthy volunteers aged 23-55 (average age $30 \mathrm{yr}$; 15 males; body mass $71.23 \pm 10.28 \mathrm{~kg}$ ) participated. All participants were screened by a physical therapist to ensure the ability to participate safely. Limb preference for kicking a ball the greatest possible distance was determined. All procedures performed were in accordance with the ethical standards of the institutional and/or national research committee and with the 1964 Helsinki declaration and its later amendments or comparable ethical standards. The Institutional Review Board of the Health Sciences Campus of the University of Southern California approved this study. Written informed consent was obtained from all participants.

\section{Task}

Typical self-selected bipedal hopping rate in humans has been reported as $2.0-2.3 \mathrm{~Hz}$ [5]. Pilot testing in our laboratory demonstrated self-selected unipedal hopping rates of 2.1-2.4 Hz. Preferred rate differed within-participant between hopping bouts and between days of testing. Participants in this study were tested at $2.3 \mathrm{~Hz}$ and $1.7 \mathrm{~Hz}$, providing notable separation between the typical and slow rates examined. All participants reported that $2.3 \mathrm{~Hz}$ hopping was easy to maintain and considerably easier to perform than $1.7 \mathrm{~Hz}$ hopping. Hopping at $1.7 \mathrm{~Hz}$ posed a significant challenge to all participants, but still allowed consistent and uninterrupted hopping. Hopping rate was prescribed by music that had a strong bass-beat at $140 \mathrm{bpm}$ for $2.3 \mathrm{~Hz}$ hopping and $100 \mathrm{bpm}$ for $1.7 \mathrm{~Hz}$ hopping. All participants were able to remain within $\pm 0.1 \mathrm{~Hz}$ of the prescribed hopping rate throughout all trials as measured by individual hop durations. Hop height was not explicitly controlled. However, hop height consistency was expected, given the rate constraint and observation of an implicit constraint of consistent limb stiffness [5].

\section{Biomechanical instrumentation}

Participants wore their own athletic shoes and attire, and were outfitted with reflective markers over the following anatomical landmarks: iliac crests, anterior superior iliac spines, space between the L5 and S1 spinous processes, greater trochanters, medial and lateral femoral epicondyles, medial and lateral malleoli, 1st and 5th metatarsal heads, and the distal pha- lanx of the pedal 2 nd rays. Additional rigid reflective marker clusters were placed bilaterally on the lateral surfaces of the thighs, shanks, and heels. 3D kinematic data were collected using an 11-camera motion analysis system (sampling rate: $250 \mathrm{~Hz}$; Qualisys $\mathrm{AB}$, Gothenburg Sweden). Ground reaction force data were collected from a $120 \mathrm{~cm} \times 120 \mathrm{~cm}$ force plate embedded in the laboratory floor (sampling rate: $1500 \mathrm{~Hz}$; AMTI Corp., Newton MA, USA).

\section{Experimental protocol}

While outfitted with reflective markers as above, each participant completed a standing static trial followed by a series of unipedal hopping trials. Upper extremity movement during hopping trials was constrained by the participants holding a $0.3 \mathrm{~kg}$ dowel across their shoulders. Participants performed a minimum of 27 consecutive hops on each lower extremity at $1.7 \mathrm{~Hz}$ and $2.3 \mathrm{~Hz}$. The order of limb testing and hopping rates was randomized. In each case, the participant was instructed to "please hop in place to the beat". A familiarization trial was performed at each hopping rate. Rest breaks of at least 1.5 minutes were given between hopping trials.

\section{Data reduction and analysis}

The first and last pair of hops from each trial were excluded from analysis. All remaining hops were qualitatively screened for visibly aberrant kinematics by reviewing video footage from the data collection. Visibly aberrant kinematics included notable trunk lean (forward or lateral), letting go of the arm-constraining dowel, or flailing the non-hopping leg. No aberrant kinematics were found in any of the included hopping trials. The accepted hops (23-28 hops per limb per participant) were parsed into stance (ground reaction force $\geq 20 \mathrm{~N}$ ) and flight (ground reaction force $<20 \mathrm{~N}$ ) phases. Only data from the stance phase were analyzed, as control of limb length during flight is not an expected neuromotor control system goal. Stance phase data were normalized to 100 frames for UCM analysis, which requires all trials to contain the same number of data points. Preliminary data exploration for the individual measures at the two hopping rates demonstrated no significant difference between the preferred kicking and contralateral limbs at either hopping rate; therefore, limbs were pooled bilaterally for a total of 46-56 hops analyzed per participant.

Kinematic data were filtered with a bidirectional 4th order Butterworth low-pass filter with cutoff frequency of $12 \mathrm{~Hz}$. Movement out of the sagittal plane was found to be minimal, with the sagittal plane pro- 
jection of the foot, shank, and thigh segment lengths differing from the segment lengths computed from 3D data in the static trial by $\leq 2 \%$ at any given time point. Therefore, the segment lengths computed from the static trial were used during all further calculations.

The exact UCM calculation methods employed in this study have been detailed elsewhere [8]. Briefly, the referent joint configuration vector $(\boldsymbol{\theta})$ was calculated at each percent of stance by averaging the local jointcoordinate sagittal foot-to-floor, and ankle and knee intersegmental angles across trials. A forward kinematic model linked changes in elemental variables (sagittal plane foot-to-floor, and ankle and knee intersegmental angles) to the task-level variable of vertical limb length (Fig. 1). Custom MATLAB code was used to compute the Jacobian matrix $(\mathbf{J}(\boldsymbol{\theta}))$ for each $1 \%$ of stance. $\mathbf{J}(\boldsymbol{\theta})$ determines how small deviations in the angles from the average configuration influence the vertical limb length. A consistent time-dependent vertical limb length was considered stable performance. The null space of $\mathbf{J}(\boldsymbol{\theta})$ is the linear approximation of the UCM subspace; variance within the UCM subspace is performance-irrelevant variability $\left(V_{\mathrm{UCM}}\right)$. Variance within the subspace orthogonal to the UCM is the performance-destabilizing variability $\left(V_{\mathrm{ORT}}\right)$. The index of motor abundance (IMA) was computed as the normalized difference between $V_{\mathrm{UCM}}$ and $V_{\mathrm{ORT}}$. IMA is also commonly referred to as the index of synergy $(\Delta V)$ in the UCM literature. $\mathrm{J}(\boldsymbol{\theta})$, $V_{\mathrm{UCM}}, V_{\mathrm{ORT}}$ and IMA were calculated at every $1 \%$ of the stance phase. The stance phase integral of each measure was then computed.

$2.3 \mathrm{~Hz}$

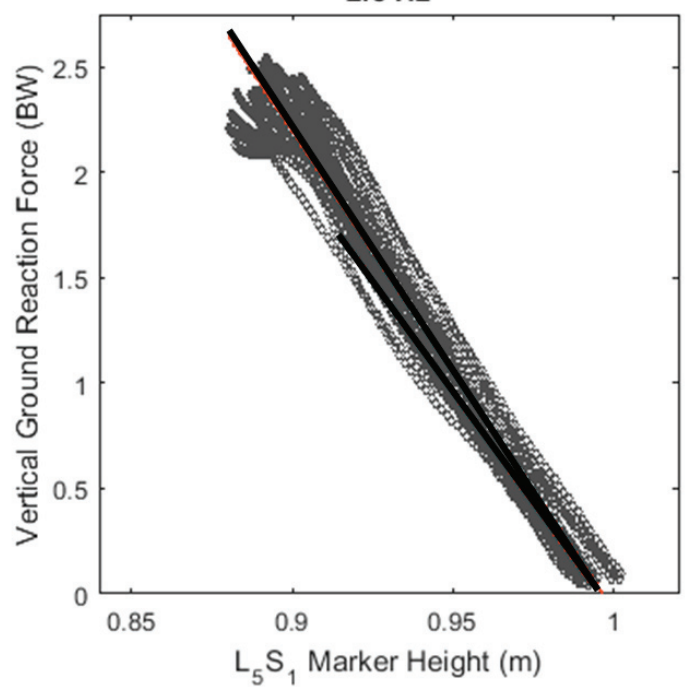

(a)

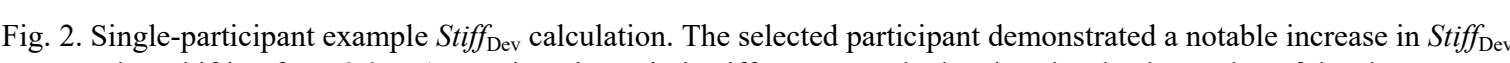
when shifting from 2.3 to $1.7 \mathrm{~Hz}$ hopping. Limb stiffness was calculated as the absolute value of the slope of the regression line fitted to the scatter plot of bodyweight-normalized vertical ground reaction force

vs. $\mathrm{L}_{5} \mathrm{~S}_{1}$ marker position. Lines overlaying the scatter plot represent stiffness during the first $50 \%$, and throughout $100 \%$, of the absorption subphase at $2.3 \mathrm{~Hz}(\mathrm{a})$ and $1.7 \mathrm{~Hz}(\mathrm{~b})$

Vertical limb length standard deviation magnitude $\left(V L L_{\mathrm{SD}}\right)$, was computed for comparison to uncontrolled manifold analysis. Vertical limb length was considered the distance from the floor to the hip joint center throughout stance. $V L L_{\mathrm{SD}}$ was calculated for each $1 \%$ of the stance phase, and then the stance phase integral was calculated.

Limb stiffness was calculated as the absolute value of the slope of the regression line fitted to the scatter plot of vertical ground reaction force (multiples of bodyweight, BW) vs. center of mass height (estimated by $\mathrm{L}_{5} \mathrm{~S}_{1}$ marker position) during the absorption subphase of stance (touchdown to center of mass minimum) [6]. Since the spring-mass model predicts linear limb stiffness, we quantified deviation from springmass model-type behavior in terms of deviation from linear limb stiffness (Stiff $f_{\text {Dev }}$ ) for both hopping rates (Fig. 2). Since there is no established method for quantifying such deviation, we calculated Stiff $_{\text {Dev }}$ as a single percentage according to Eq. (1). The larger the negative ratio, the greater the deviation from springmass model-type behavior. Specifically, a larger negative ratio represents a greater decrement in limb stiffness late in the absorption sub-phase, compared to the initial limb stiffness following touchdown.

$$
\text { Stiff }_{\text {Dev }}=
$$

Stiffness $_{100 \% \text { absorption sub-phase }}-$ Stiffness $_{\text {First } 50 \% \text { absorption sub-phase }}$

$$
\text { Stiffness } S_{\text {First } 50 \% \text { absorption sub-phase }}
$$

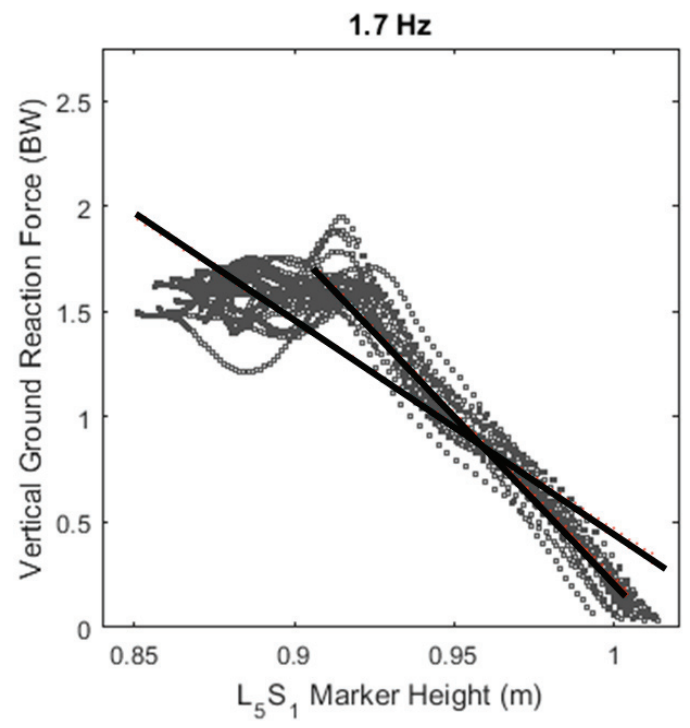

(b) 


\section{Statistical analyses}

SPSS Statistics 22.0 software (IBM Corp.; Armonk NY, USA) was used for all statistical analyses. Differences for each measure between $2.3 \mathrm{~Hz}$ and $1.7 \mathrm{~Hz}$ hopping conditions were determined with pairedsamples $t$-tests. Data were reported as mean \pm standard deviation. Cohen's D effect sizes were calculated for all paired $t$-test results. Pearson correlations were used to examine the relationship between the change scores (Value ${ }_{1.7 \mathrm{~Hz}}-$ Value $_{2.3 \mathrm{~Hz}}$ ) for Stiff Dev $_{\text {and the two }}$ variability measures (total stance phase IMA and total stance phase $\left.V L L_{\mathrm{SD}}\right)$. Significance was set at $\alpha=0.05$.

\section{Results}

Limb stiffness was greater during $2.3 \mathrm{~Hz}$ than $1.7 \mathrm{~Hz}$ hopping (Table 1, Fig. 3). All participants demonstrated decreased stiffness when switching from typi-

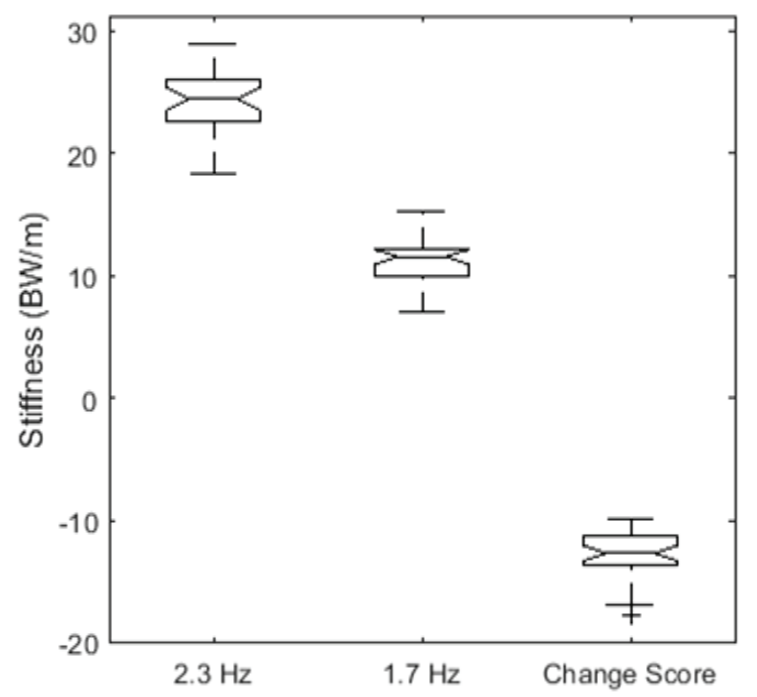

Fig. 3. Limb stiffness. Limb stiffness decreased with the shift from 2.3 to $1.7 \mathrm{~Hz}$ hopping cal $(2.3 \mathrm{~Hz})$ to slow $(1.7 \mathrm{~Hz})$ hopping $(95 \% \mathrm{CI}$ : decrease of $12.20-13.56 \mathrm{BW} / \mathrm{m})$. Deviation from spring-mass model-type behavior was greater during $1.7 \mathrm{~Hz}$ than $2.3 \mathrm{~Hz}$ hopping; this is represented by a more negative Stiff $_{\text {Dev }}$ (Table 1, Fig. 4). All participants demonstrated increased Stiff ${ }_{\text {Dev }}$ when switching from typical to slow hopping (95\% CI: more negative by $0.25-0.33$ ).

Total stance phase standard deviation of vertical limb length $\left(V L L_{\mathrm{SD}}\right)$ was greater during $1.7 \mathrm{~Hz}$ than $2.3 \mathrm{~Hz}$ hopping (Table 1, Fig. 5). Most participants $(85 \%)$ demonstrated increased $V L L_{\mathrm{SD}}$ when switching from typical to slow hopping $(95 \% \mathrm{CI}$ : increase of $0.11-0.27 \mathrm{~m}$ ).

Total stance phase index of motor abundance regarding limb length stabilization (IMA) was greater during $2.3 \mathrm{~Hz}$ than $1.7 \mathrm{~Hz}$ hopping (Table 1, Fig. 6a). IMA quantifies the degree to which a greater or lesser proportion of the total variability is channeled into the performance-irrelevant $\left(V_{\mathrm{UCM}}\right)$ vs. the performancedestabilizing $\left(V_{\mathrm{ORT}}\right)$ subspace. A larger positive IMA

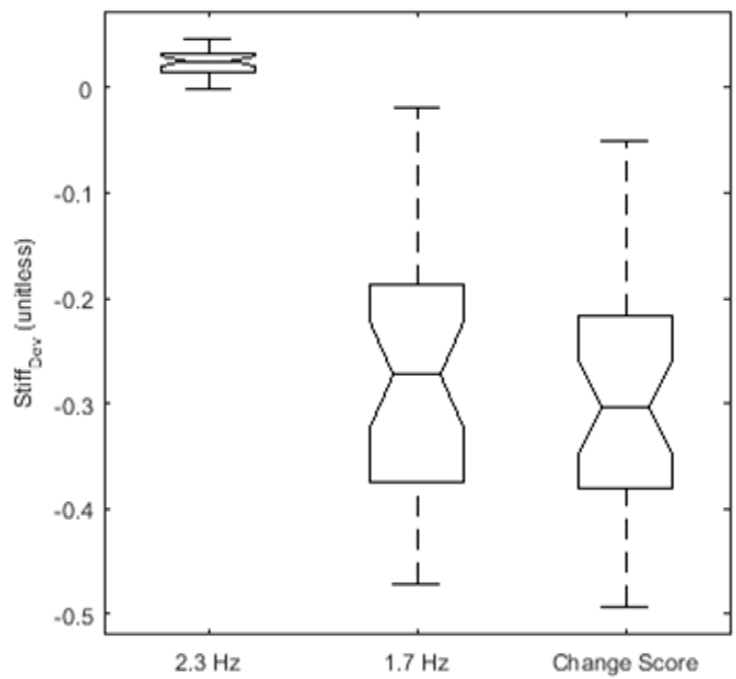

Fig. 4. Deviation from spring-mass model-type behavior. Stiff $_{\text {Dev }}$ became more negative with the shift from 2.3 to $1.7 \mathrm{~Hz}$ hopping

Table 1. Results of paired $t$-tests for the difference between hopping at $2.3 \mathrm{~Hz}$ and $1.7 \mathrm{~Hz}$

\begin{tabular}{|c|c|c|c|c|}
\hline & \multirow{2}{*}{$\begin{array}{c}2.3 \mathrm{~Hz} \\
\text { Average (SD) }\end{array}$} & \multirow{2}{*}{$\frac{1.7 \mathrm{~Hz}}{\text { Average (SD) }}$} & \multicolumn{2}{|c|}{ Difference between 2.3 and $1.7 \mathrm{~Hz}$} \\
\hline & & & $p$-value & Effect size \\
\hline Limb Stiffness [BW/m] & $24.17(2.51)$ & $11.29(1.58)$ & $<0.001$ & 6.639 \\
\hline Stiff $f_{\text {Dev }}[\%]$ & $0.02(0.01)$ & $-0.26(0.13)$ & $<0.001$ & 2.355 \\
\hline$V L L_{\mathrm{SD}}[\mathrm{m}]$ & $0.87(0.24)$ & $1.06(0.24)$ & $<0.001$ & 0.863 \\
\hline IMA [-] & $60.15(19.30)$ & $54.64(16.04)$ & 0.35 & 0.377 \\
\hline$V_{\mathrm{UCM}}\left[\mathrm{rad}^{2} / \mathrm{DOF}\right]$ & $0.24(0.07)$ & $0.30(0.14)$ & 0.003 & 0.551 \\
\hline$V_{\mathrm{ORT}}\left[\mathrm{rad}^{2} / \mathrm{DOF}\right]$ & $0.14(0.06)$ & $0.19(0.12)$ & 0.005 & 0.517 \\
\hline
\end{tabular}




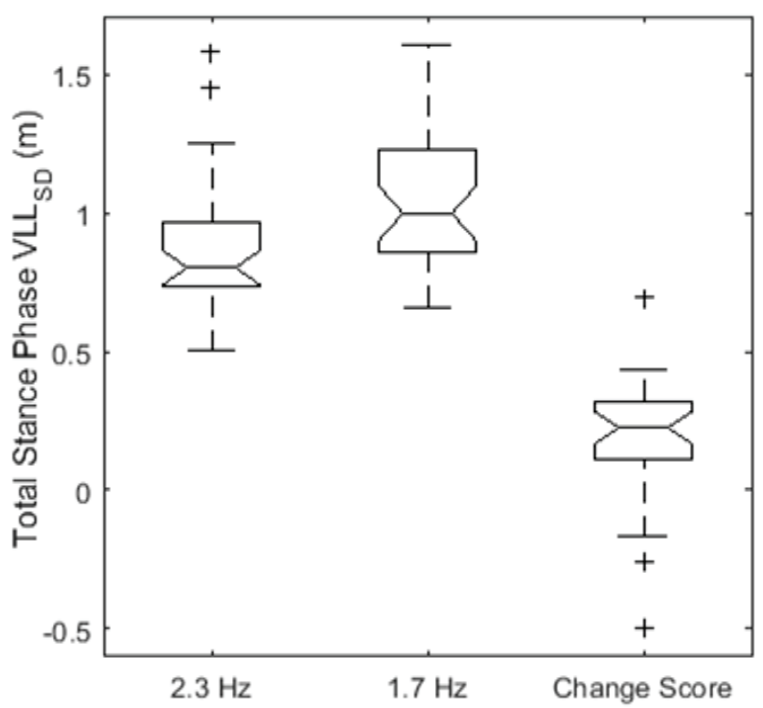

Fig. 5. Total stance phase variability in vertical limb length $\left(V L L_{\mathrm{SD}}\right)$. $V L L_{\mathrm{SD}}$ increased with the shift from 2.3 to $1.7 \mathrm{~Hz}$ hopping

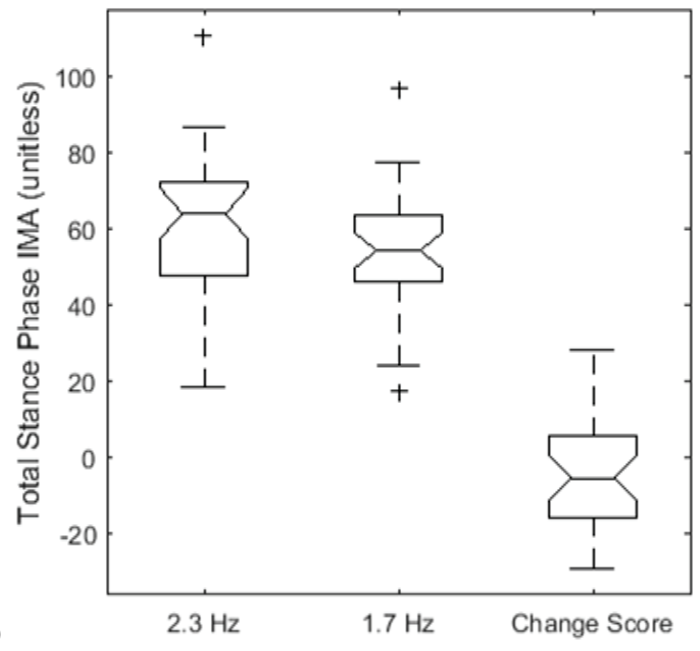

(a)

$\begin{array}{lll}2.3 \mathrm{~Hz} & 1.7 \mathrm{~Hz} \quad \text { Change Score }\end{array}$ value at a given time-point indicates $V_{\mathrm{UCM}}>V_{\mathrm{ORT}}$, so that most of the variability that occurs still allows a consistent vertical limb length across hopping repetitions. An IMA value near zero indicates that $V_{\mathrm{UCM}}$ $\approx V_{\mathrm{ORT}}$, so that vertical limb length is not particularly consistent across hopping repetitions. The majority of participants (68\%) decreased IMA when switching from typical to slow hopping (95\% CI: decrease of 0.41-10.59).

Change in IMA is dictated by changes in its determinants $\left(V_{\mathrm{UCM}}\right.$ and $\left.V_{\mathrm{ORT}}\right)$. Both $V_{\mathrm{UCM}}$ and $V_{\mathrm{ORT}}$ were greater during $1.7 \mathrm{~Hz}$ than $2.3 \mathrm{~Hz}$ hopping (Table 1, Figs. $6 \mathrm{~b}$ and $6 \mathrm{c})$. Most participants $(74 \%)$ increased $V_{\mathrm{UCM}}$ when switching from typical to slow hopping (95\% CI: increase of $\left.0.03-0.11 \mathrm{rad}^{2} / \mathrm{DOF}\right)$. Most participants $(74 \%)$ also increased $V_{\text {ORT }}$ when switching from typical to slow hopping $(95 \% \mathrm{CI}$ : increase of $\left.0.02-0.08 \mathrm{rad}^{2} / \mathrm{DOF}\right)$.

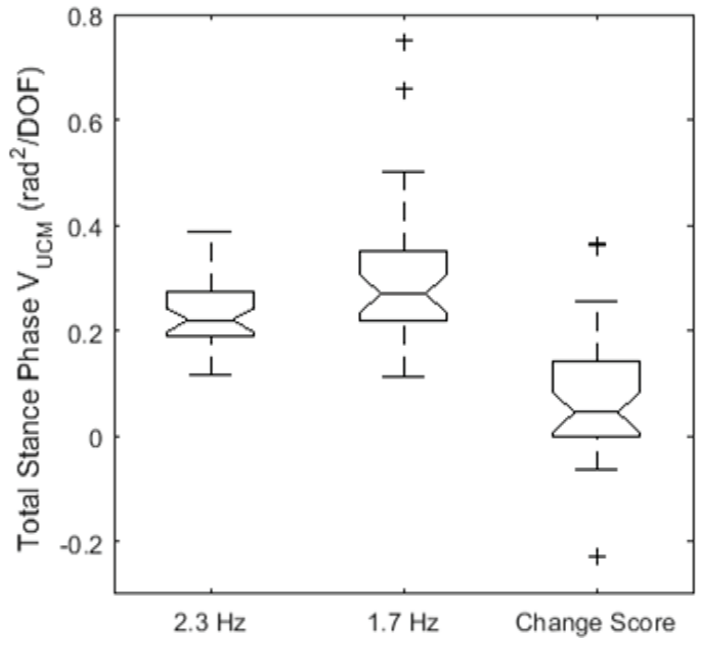

(b)

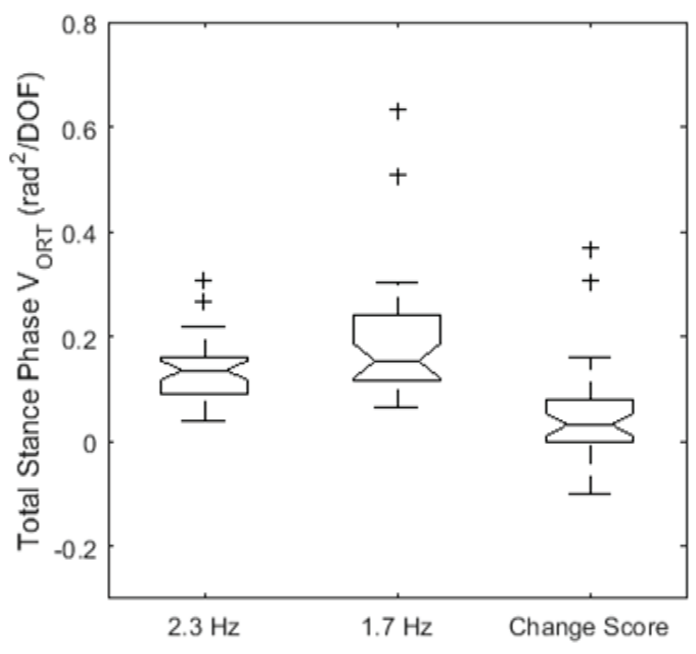

Fig. 6. Total stance phase index of motor abundance regarding limb length control (IMA) and its determinants $\left(V_{\mathrm{UCM}}\right.$ and $\left.V_{\mathrm{ORT}}\right)$ :

(a) IMA decreased with the shift from 2.3 to $1.7 \mathrm{~Hz}$ hopping, (b) $V_{\mathrm{UCM}}$ increased with the shift from 2.3 to $1.7 \mathrm{~Hz}$ hopping,

(c) $V_{\text {ORT }}$ increased with the shift from 2.3 to $1.7 \mathrm{~Hz}$ hopping 

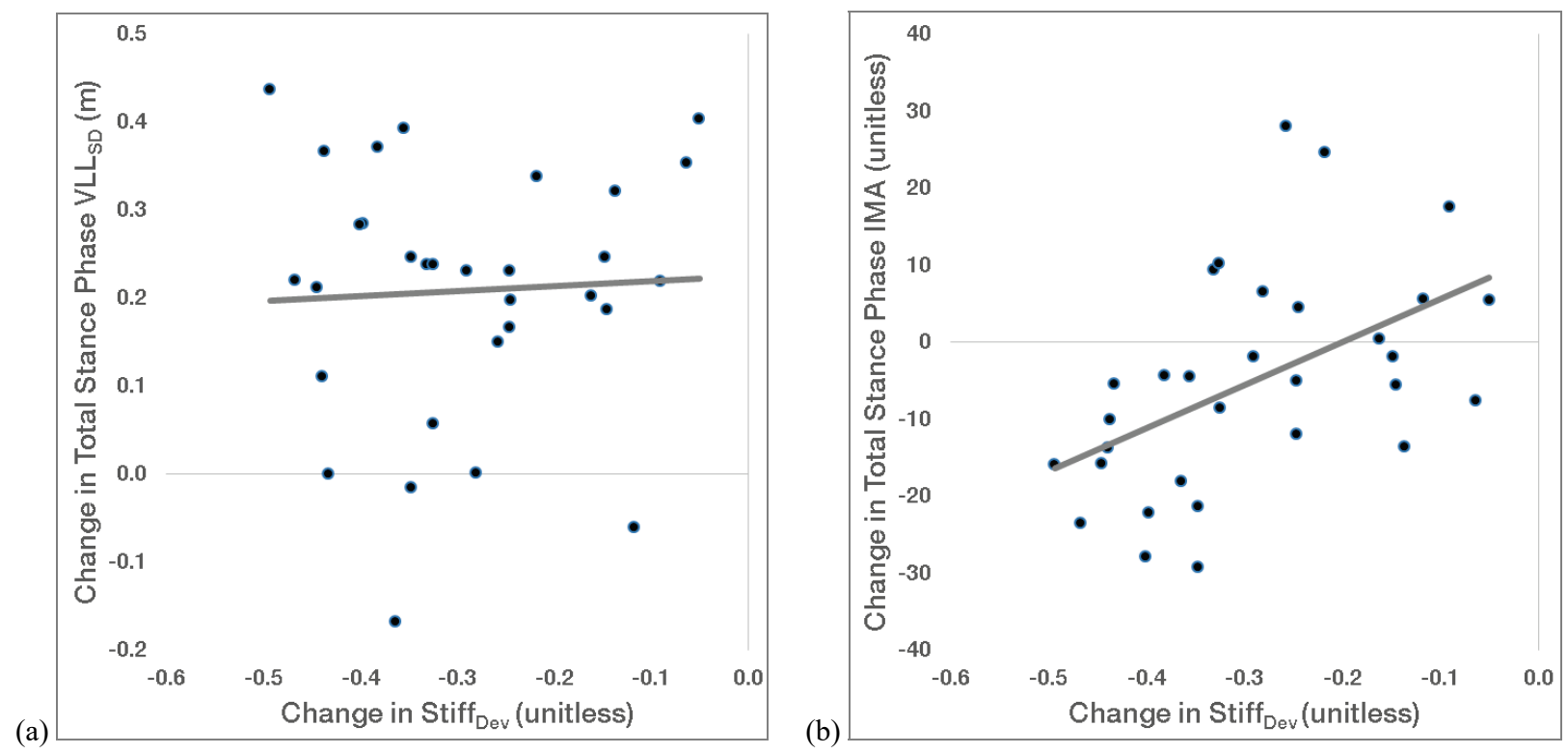

Fig. 7. Correlation between deviation from spring-mass model-type behavior and variability measures:

(a) there was no significant relationship between change scores for Stiff $f_{\mathrm{Dev}}$ and $V L L_{\mathrm{SD}}$,

(b) there was a moderate correlation between change scores for Stiff Dev $_{\text {and IMA }}$

Change scores for Stiff $_{\mathrm{Dev}}$ and $V L L_{\mathrm{SD}}$ did not correlate ( $p=0.755, R=-0.056)$. The lack of relationship held when the 3 outlier participants' data was removed ( $p=0.784, R=0.051$, Fig. 7a). In contrast, the change scores for StiffDev and IMA were moderately correlated ( $p=0.003, R=0.489$ ). This relationship improved in strength when the 3 outlier participants' data was removed ( $p=0.004, R=0.502$, Fig. $7 \mathrm{~b})$.

\section{Discussion}

Two different methods of measuring movement variability that provide limb length (task level) control information, within the same data set, were compared. The first method was the basic movement variability measure of $V L L_{\mathrm{SD}}$. The second method was UCM analysis of the degree to which coordination between variability in foot, shank and thigh positioning contributed to stabilization of vertical limb length (IMA). The capacity of $V L L_{\mathrm{SD}}$ and IMA to illuminate the neuromotor control system's response (Stiff $f_{\text {Dev }}$ ) to the perturbation of switching from typical $(2.3 \mathrm{~Hz})$ to challengingly slow $(1.7 \mathrm{~Hz})$ hopping was determined. Correlations between change scores for $V L L_{\mathrm{SD}}$ and IMA with change in Stiff $f_{\text {Dev }}$ were examined. Change scores for IMA and Stiff $f_{\text {Dev }}$ were moderately correlated, but no relationship was found between $V L L_{\mathrm{SD}}$ and Stiff $f_{\text {Dev }}$ change scores (Fig. 7).

Limb stiffness is closely related to the control of limb length, and decreased limb stiffness with the 2.3 to $1.7 \mathrm{~Hz}$ shift found in this study (Fig. 3) corroborates previous findings [5]. Previous studies show that hopping at rates slower than typically self-selected challenges spring-mass model-type behavior (particularly linearity of limb stiffness) [5]. For all participants, limb stiffness was nearly linear throughout all of stance at $2.3 \mathrm{~Hz}$. Similarly, for all participants, limb stiffness was linear throughout the majority of stance at $1.7 \mathrm{~Hz}$, likely indicating an attempt to maintain spring-mass model-type behavior even in this challengingly slow condition, which could have warranted a very different control strategy. Deviation from linearity occurred only near mid-stance, where participants demonstrated relatively lesser stiffness. The greater deviation from spring-mass model-type behavior (more negative Stiff $_{\text {Dev }}$ ) with the shift from 2.3 to $1.7 \mathrm{~Hz}$ found in this study (Fig. 4) matches these previous findings.

UCM analysis considers variability at two levels to address both magnitude and quality of movement variability. We examined how variability in lower extremity segmental postures either contributed to $\left(V_{\text {ORT }}\right)$ or minimized $\left(V_{\mathrm{UCM}}\right)$ variability of overall vertical limb length across hopping trials. The index of motor abundance (IMA) expresses the degree to which a greater or lesser proportion of the total variability is shunted into the vertical limb length-irrelevant $\left(V_{\mathrm{UCM}}\right)$ type, as opposed to vertical limb length-destabilizing $\left(V_{\mathrm{ORT}}\right)$ type. In the present study, $68 \%$ of participants decreased IMA with increasing task difficulty, while $32 \%$ increased IMA (Fig. 6). Previous studies have 
demonstrated alterations in IMA with perturbations or changes in task difficulty [1], [22]. Despite small sample sizes $(6 \leq n \leq 11)$, neither of these studies discussed individual IMA-responses to the task changes probed.

To understand what drives changes in IMA, individual changes in $\mathrm{V}_{\mathrm{UCM}}$ and $\mathrm{V}_{\mathrm{ORT}}$ variability types must be examined. This need is underscored by the fact that participants in this study who decreased IMA with increasing task difficulty were not the exact same subset of participants who decreased $V L L_{\mathrm{SD}}$. Of the 23 participants who decreased IMA with the shift from 2.3 to $1.7 \mathrm{~Hz}, 74 \%$ did so by increasing total variability $\left(V_{\mathrm{ORT}}\right.$ more than $\left.V_{\mathrm{UCM}}\right), 13 \%$ by decreasing total variability ( $V_{\mathrm{UCM}}$ more than $V_{\mathrm{ORT}}$ ), and $13 \%$ by increasing $V_{\mathrm{ORT}}$ while decreasing $V_{\mathrm{UCM}}$. Increased total variability matches with some previous UCM studies, as well as several non-UCM variability studies that demonstrated increasing variability with increasing task difficulty [2], [9], [21], [22], [24]. The $18 \%$ of participants who responded to the hopping rate shift with decreased total variability match previous non-UCM variability studies demonstrating decreasing variability with increasing task difficulty [9], [24].

Vertical limb length standard deviation $\left(V L L_{\mathrm{SD}}\right)$ is a typical task-level movement variability measure that quantifies between-hop "error" in the control system. A larger $V L L_{\mathrm{SD}}$ value may indicate poor limb length control. In this study, $85 \%$ of participants increased $V L L_{\mathrm{SD}}$ with the shift from 2.3 to $1.7 \mathrm{~Hz}$ hopping (Fig. 5); this finding is consistent with previous literature demonstrating increased error-associated variability with increasing task difficulty [1], [12], [18].

This study illustrates the capacity of UCM analysis to illuminate the neuromotor control system's response (Stiff $f_{\text {Dev }}$ ) to the perturbation of switching from typical $(2.3 \mathrm{~Hz})$ to challengingly slow $(1.7 \mathrm{~Hz})$ hopping condition. Limb stiffness is closely related to limb length control, which is captured in different manners by $V L L_{\mathrm{SD}}$ and IMA. A significant large correlation was found between change scores for IMA and Stiff $f_{\text {Dev }}$, but no relationship was found between

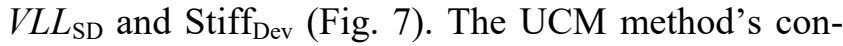
sideration of how the neuromotor control system is shunting movement variability into performance-irrelevant and performance-destabilizing subspaces rather than strictly error-based movement variability likely underlies this finding. As such, UCM-based movement variability analysis may be better at illuminating more global control aspects than the typical types of movement variability measures (e.g., $V L L_{\mathrm{SD}}$ ).

This study was limited by participant age skewing toward the younger end of the age range tested $(68 \%$ under the average age of $30 \mathrm{yr}$., $26 \%>30 \mathrm{yr}$.). However, it has been shown that young and elderly adults display similar spring-mass model-type behavior and limb stiffness across multiple hopping rates [13]. The vertical limb length control measured in this study is closely related to limb stiffness, and thus could be expected to be reasonably similar across age groups as well.

\section{Acknowledgements}

This work was partially funded by a Promotion of Doctoral Studies Scholarship from the Foundation for Physical Therapy.

\section{References}

[1] Auyang A.G., Chang Y.-H.H., Effects of a Foot Placement Constraint on Use of Motor Equivalence during Human Hopping, PLoS One, 2013, 8, e69429.

[2] Bartlett R., Movement variability and its implications for sports scientists and practitioners: an overview, Int. J. Sports Sci. Coach, 2008, 3, 113-124.

[3] Cunningham T.J., Mullineaux D.R., Noehren B., Shapiro R., UhL T.L., Coupling angle variability in healthy and patellofemoral pain runners, Clin. Biomech., 2014, 29, 317-322.

[4] Davids K., Glazier P., Araújo D., Bartlett R., Movement systems as dynamical systems, Sport Med., 2003, 33, $245-260$.

[5] Farley C.T., Blickhan R., Saito J., Taylor C.R., Hopping frequency in humans: a test of how springs set stride frequency in bouncing gaits, J. Appl. Physiol., 1991, 71, 2127-2132.

[6] FARley C.T., GonZAlez O., Leg Stiffness and Human Stride Frequency in Running, J. Biomech., 1996, 29, 181-186.

[7] Ferris D.P., Bohra Z.A., LuKos J.R., Kinnaird C.R., Neuromechanical adaptation to hopping with an elastic ankle-foot orthosis, J. Appl. Physiol., 2006, 100, 163-170.

[8] Fietzer A.L., Winstein C.J., Kulig K., Changing one's focus of attention alters the structure of movement variability, Hum. Mov. Sci., 2018, 62, 14-24.

[9] Ghanavati T., Salavati M., Karimi N., Negahban H., EBrahimi I., Mehravar M. et al., Intra-limb coordination while walking is affected by cognitive load and walking speed, J. Biomech., 2014, 47, 2300-2305.

[10] Glasgow P., Bleakley C.M., Phillips N., Being able to adapt to variable stimuli: the key driver in injury and illness prevention, Br. J. Sports Med., 2013, 47, 64-65.

[11] Hamill J., Palmer C., Van Emmerik R.E.A., Coordinative variability and overuse injury, Sport Med. Arthrosc. Rehabil. Ther. Technol., 2012, 4, 45-53.

[12] Hiley M.J., Zuevsky V.V., Yeadon M.R., Is skilled technique characterized by high or low variability? An analysis of high bar giant circles, Hum. Mov. Sci., 2013, 32, 171-180.

[13] Hobara H., Kobayashi Y., Yoshida E., Mochimaru M., Leg stiffness of older and younger individuals over a range of hopping frequencies, J. Electromyogr. Kinesiol., 2015, 25, 305-309. 
[14] James C.R., DufEK J.S., BATES B.T., Effects of injury proneness and task difficulty on joint kinetic variability, Med. Sci. Sports Exerc., 2000, 32, 1833-1844.

[15] KapUR S., Zatsiorsky V.M., LATASH M.L., Age-related changes in the control of finger force vectors, J. Appl. Physiol., 2010, 109, 1827-1841.

[16] LATASH M.L., The bliss (not the problem) of motor abundance (not redundancy), Exp. Brain Res., 2012, 217, 1-5.

[17] Moritz C.T., FARLEY C.T., Human hopping on very soft elastic surfaces: implications for muscle pre-stretch and elastic energy storage in locomotion, J. Exp. Biol., 2005, 208, 939-949.

[18] Mullineaux D.R., Uhl T.L., Coordination-variability and kinematics of misses versus swishes of basketball free throws, J. Sports Sci., 2010, 28, 1017-1024.

[19] Newell K.M., Broderick M.P., Deutsch K.M., Slifkin A.B., Task goals and change in dynamical degrees of freedom with motor learning, J. Exp. Psychol. Hum. Percept. Perform., 2003, 29, 379-387.

[20] Park J., Jo H.J., Lewis M.M., HuAnG X., Latash M.L., Effects of Parkinson's disease on optimization and structure of variance in multi-finger tasks, Exp. Brain Res., 2013, 231, 51-63.

[21] Reisman D.S., SchOlz J.P., SchÖNER G., Coordination underlying the control of whole body momentum during sit-to-stand, Gait Posture, 2002, 15, 45-55.

[22] Scholz J.P., REISMAN D., SCHÖNER G., Effects of varying task constraints on solutions to joint coordination in a sit-to-stand task, Exp. Brain Res., 2001, 141, 485-500.

[23] SchOlz J.P., SchÖNER G., The uncontrolled manifold concept: Identifying control variables for a functional task, Exp. Brain Res., 1999, 126, 289-306.

[24] SeAY J.F., VAN EMmeriK R.E.A., Hamill J., Low back pain status affects pelvis-trunk coordination and variability during walking and running, Clin. Biomech., 2011, 26, 572-578. 